\title{
Microplastics as Vectors for Environmental Contaminants : Exploring Sorption, Desorption, and Transfer to Biota
}

Hartmann, Nanna B.; Rist, Sinja; Bodin, Julia; Jensen, Louise Helene Søgaard; Nørgaard Schmidt, Stine; Mayer, Philipp; Meibom, Anders; Baun, Anders

Published in:

Integrated Environmental Assessment and Management

Link to article, DOI:

10.1002/ieam.1904

Publication date:

2017

Document Version

Peer reviewed version

Link back to DTU Orbit

Citation (APA):

Hartmann, N. B., Rist, S., Bodin, J., Jensen, L. H. S., Nørgaard Schmidt, S., Mayer, P., Meibom, A., \& Baun, A. (2017). Microplastics as Vectors for Environmental Contaminants : Exploring Sorption, Desorption, and Transfer to Biota. Integrated Environmental Assessment and Management, 13(3), 488-493.

https://doi.org/10.1002/ieam.1904

\section{General rights}

Copyright and moral rights for the publications made accessible in the public portal are retained by the authors and/or other copyright owners and it is a condition of accessing publications that users recognise and abide by the legal requirements associated with these rights.

- Users may download and print one copy of any publication from the public portal for the purpose of private study or research.

- You may not further distribute the material or use it for any profit-making activity or commercial gain

- You may freely distribute the URL identifying the publication in the public portal 


\section{Microplastics as vectors for environmental contaminants: Exploring sorption, desorption, and transfer to biota}

Accepted for publication in: Integrated Environmental Assessment and Management DOI:10.1002/ieam.1904

Nanna Bloch Hartmann ${ }^{\dagger}{ }^{*}$, Sinja Rist ${ }^{\dagger}$, Julia Bodin ${ }^{\ddagger}$, Louise Helene Søgaard Jensen ${ }^{\ddagger}$, Stine Nørgaard Schmidt ${ }^{\dagger}$, Philipp Mayer $^{\dagger}$, Anders Meibom ${ }^{\ddagger}$, Anders Baun ${ }^{\dagger}$

${ }^{\dagger}$ Technical University of Denmark, Department of Environmental Engineering, Bygningstorvet B115, DK-2800 Kgs Lyngby, Denmark

${ }^{\ddagger}$ Laboratory for Biological Geochemistry, School of Architecture, Civil and Environmental Engineering at Ecole Polytechnique Fédérale de Lausanne (EPFL), CH-1015 Lausanne, Switzerland.

* corresponding author, nibh@env.dtu.dk 
Microplastics as contaminant vectors - exploring the processes - Postprint version

\begin{abstract}
The occurrence and effects of microplastics (MPs) in the aquatic environment are receiving increasing attention. In addition to possible direct adverse effects on biota, their potential role as vectors for hydrophobic organic chemicals (HOCs), compared to natural pathways, is a topic of much debate. It is evident, however, that temporal and spatial variations of MP occurrence do (and will) occur. To further improve the estimations of the role of MPs as vectors for HOC transfer into biota under varying MP concentrations and environmental conditions, it is important to identify and understand the governing processes. Here, we explore HOC sorption to and desorption from MPs and the underlying principles for their interactions. We discuss intrinsic and extrinsic parameters influencing these processes, and focus on the importance of the exposure route for diffusive mass transfer. Also, we outline research needed to fill knowledge gaps and improve model-based calculations of MP-facilitated HOC transfer in the environment.
\end{abstract}

\title{
Keywords
}

Microplastics, contaminants, hydrophobic organic chemicals (HOCs), exposure, transfer, ecotoxicity 
Microplastics as contaminant vectors - exploring the processes - Postprint version

\section{INTRODUCTION}

Interactions between microplastics (MPs) and organic contaminants take place before, during, and after their release to natural environments. These interactions occur as a result of product formulation (e.g., by addition of plasticisers and preservatives) as well as unintentionally, in e.g., wastewater, urban runoff, and landfill leachate containing complex mixtures of other environmental contaminants. Recent studies have demonstrated the ability of MPs to carry environmental contaminants (e.g. Rochman et al. 2013; Velzeboer et al. 2014). This has led to the hypothesis that, in addition to direct effects of interactions with biota, MPs may also play a role in aquatic ecotoxicology as vectors for toxic substances.

The role of MPs as contaminant vectors has been the topic of experimental studies and review papers, both supporting and challenging this hypothesis. Some argue that plastic debris and HOCs form complex cocktails that increase the overall bioavailability of HOCs to aquatic organisms and ultimately to humans (Vethaak and Leslie 2016). This hypothesis has been challenged with the argument that plastics play a minor role as contaminant vectors compared to natural particles, such as suspended organic particulates and natural prey, because of their relatively low abundance in the environment (Koelmans et al. 2016). Based on data from published studies, calculations have been made to assess the overall relative importance of MPs as contaminant vectors compared to other naturally occurring sorbents (Koelmans et al. 2016; Bakir et al. 2016). Models of increasing complexity have been developed and applied, taking into account the partition ratios between solid phases (including plastic) and water, MP age distribution, the relative abundance and ingested amounts of MPs (Koelmans et al. 2016) as well as the role of gut surfactants, pH, and temperature on HOC desorption (Bakir et al. 2016). With increasing plastic production and use in society, environmental occurrence of MPs will inevitable increase in the future (temporal increase) and also vary depending on specific local-scale conditions (spatial variations). Expanding our fundamental understanding of the processes involved in MP facilitated transport of contaminants is therefore 
Microplastics as contaminant vectors - exploring the processes - Postprint version

necessary, allowing us to develop more accurate models and thereby evaluate the role of this process under varying and case-specific conditions.

A conceptual overview of the topics covered in this paper is illustrated in Figure 1. In the following sections, we describe the processes governing the interactions between MPs and HOCs and their subsequent uptake into aquatic organisms, including HOC sorption to and desorption from MPs and parameters affecting HOC distribution. We discuss the impact of different uptake routes on MP-facilitated HOC transfer into biota, including direct MP contact exposure (internal and external). Finally, we point to knowledge gaps and research required for a more comprehensive understanding and modelling of these processes.

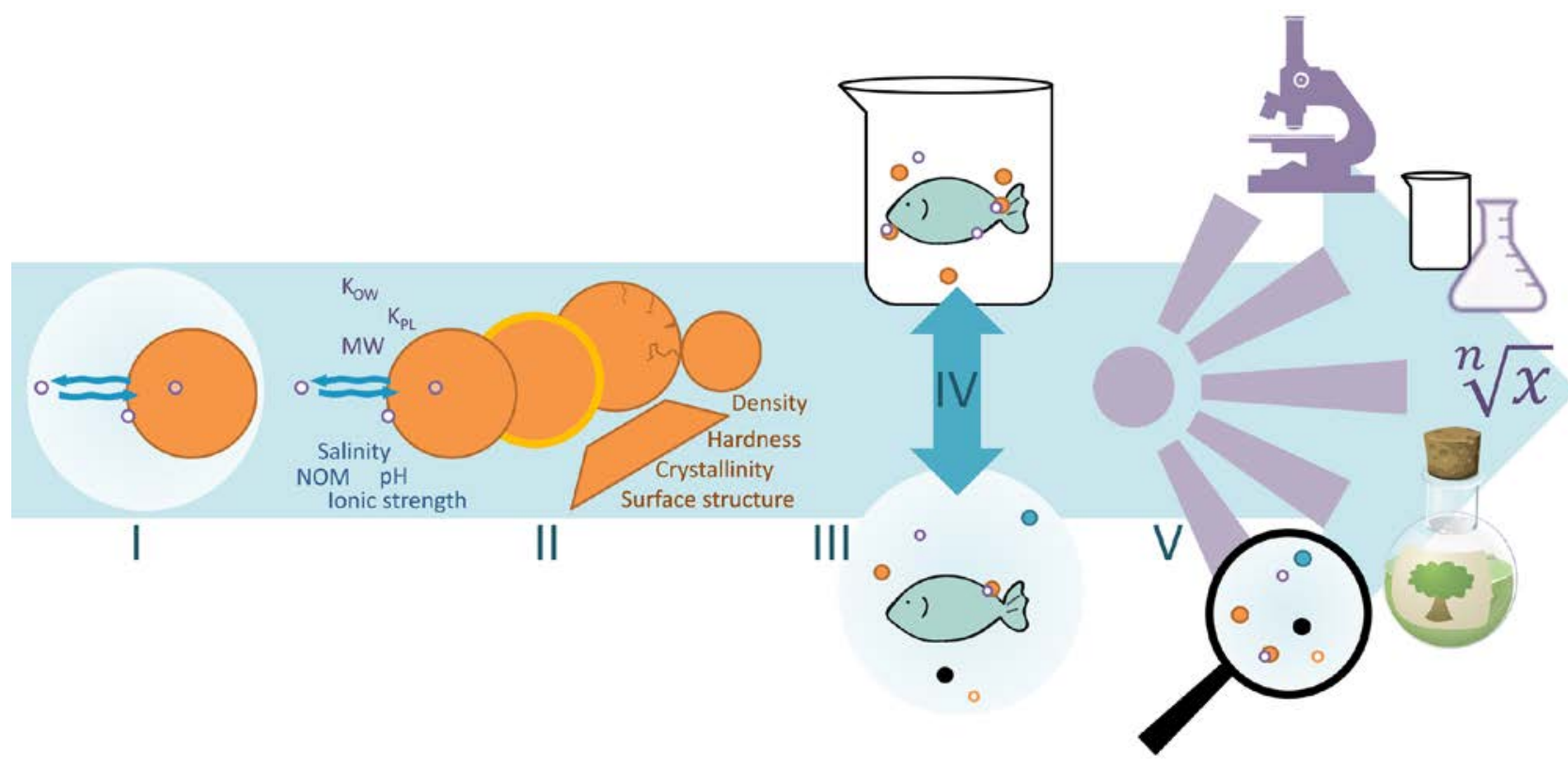

Figure 1. Overview of the topics covered in this paper: I) HOC sorption to and desorption from MPs; II) The influence of intrinsic and extrinsic properties on HOC distribution to MPs; III) Transfer of HOCs between MPs and biota, including direct contact transfer; IV) The role of in situ and laboratory studies when exploring these processes; and, V) Outlook on knowledge gaps and future research directions 


\section{SORPTION AND DESORPTION - THE INFLUENCE OF INTRINSIC AND EXTRINSIC PROPERTIES}

Due to their hydrophobicity and lipophilicity, HOCs sorb to non-polar phases in natural aquatic environments, including sediment particles, suspended organic matter, and MPs. The distribution of HOCs between different types of particulate matter and the aqueous phase is a function of the intrinsic properties of the given HOC as well as the particulate matter (under given environmental conditions). The distribution is governed by equilibrium partitioning and molecular diffusion and quantified by plastic- and HOC-specific partition ratios (Mayer et al. 2000; O’Connor et al. 2016).

Overall, the sorption of a compound to a sorbent can take place by absorption or by adsorption. Absorption involves the partitioning of molecules into a sorbing matrix, keeping the molecules dissolved and retained only by relatively weak van-der-Waals forces. Such partitioning is largely driven by the hydrophobicity of the compound, "pushing” it out of the water and into the matrix, leading to partition ratios that, for HOCs and polymers, generally correlate well with their octanol to water partition ratios (Mayer et al. 2000; O’Connor et al. 2016). On the contrary, surface adsorption can involve a wide range of different interaction forces including van-der-Waals, ionic, steric, п-п interactions and covalent bonds. At low concentrations of the chemical, adsorption generally leads to much higher partition ratios compared to absorption due to the stronger interaction forces on the surface. However, at higher concentrations of the chemical, absorption often takes over as the dominant retention process due to the much larger volume to accommodate the molecules (Luthy et al. 1997; Cornelissen et al. 2005).

While this issue was initially a matter of debate within analytical chemistry (Hawthorne et al. 2000; Vaes et al. 2000), experimental studies have now demonstrated that hydrophobic organic contaminants (such as polycyclic aromatic hydrocarbons (PAHs) and polychlorinated biphenyls) 
Microplastics as contaminant vectors - exploring the processes - Postprint version

can be absorbed into various polymers, including silicone (Mayer et al. 2000) and low density polyethylene (LDPE) (Lohmann 2012). This is the mechanistic basis for most passive sampling techniques for HOCs (Booij et al. 2016). Recent tests with seven different HOCs and four polymers showed that the sorption process depends strongly on the polymer type (Hüffer and Hofmann 2016).

Transport of the HOCs within the polymer matrix depends on a number of factors, such as the free volume within the polymer and the segmental mobility of the polymer chains (Rusina et al., 2009), defined as the ability of the polymer chains to move and attain different physical conformations. This, in turn, is related to the glass transition temperature, degree of crosslinking and crystallinity of the polymer. Crystallinity is a descriptor for the polymer structure; the more ordered and fixed, the higher the crystallinity. Increasing crystallinity results in decreasing capacity and rate of absorption of a contaminant into the polymer matrix (Mato et al. 2001; Karapanagioti and Klontza 2008). Polymers often contain both crystalline and amorphous (i.e., non-crystalline) regions. The amorphous regions, where sorption of HOCs generally occurs (Teuten et al., 2009), can be either 'glassy' or 'rubbery', depending on the glass transition temperature of the polymer. Glassy amorphous polymers (e.g., polyvinyl chloride (PVC) and polystyrene (PS)) are more condensed and cross-linked than rubbery amorphous polymers (e.g., polyethylene (PE) and polypropylene (PP)); the former therefore have lower diffusivity than the latter. Furthermore, glassy polymers have internal pores ('nanovoids'), creating strong adsorption sites and contributing to slow HOC release rates (Teuten et al. 2009). Hüffer and Hoffman recently reported that for PE, a rubbery polymer, absorption dominated, whereas for the glassy polymers, PS, PVC, and polyamide, adsorption was found to be dominant (Hüffer and Hofmann 2016). These findings emphasize that sorption processes are closely linked to the polymer structure.

Another important factor that determines HOC sorption to polymers is the distance between the polymer chains. The greater the distance, the easier it is for chemicals to diffuse into/through the 
Microplastics as contaminant vectors - exploring the processes - Postprint version

matrix (Pascall et al. 2005) and the higher the sorptive capacity (Rochman et al. 2013). For this reason, for example, $\mathrm{PE}$ is expected to have a greater sorptive capacity in comparison to PP (Rochman et al. 2013). Similarly, additives can change the structure of the polymer and thus its sorption capacity (Endo et al. 2005). Surface polarity plays a role as HOCs interact more strongly with non-polar surfaces (Mato et al. 2001). MP shape and size define the surface-to-volume ratio and diffusional length scales, which in turn determine the time to reach equilibrium and the rate of absorption/desorption (Teuten et al. 2009).

Weathering of MPs, which is the integrated result of environmental conditions and exposure time, can modify their properties. Photo-weathering, which causes bond breakages in the polymer matrix and subsequent formation of cracks, increases the surface area and pore size resulting in increased diffusivity and sorption of HOCs. In contrast, reactions with oxygen can increase the surface polarity, which decreases the affinity for HOCs (Endo et al. 2005; Teuten et al. 2009). Weathering can also lead to an increase in crystallinity of the polymer (Mato et al. 2001; Karapanagioti and Klontza 2008), thereby reducing sorption of HOCs. MPs in the aquatic environment are furthermore prone to fouling, whereby biomaterials can serve as additional sorbents (Endo et al. 2005).

HOC hydrophobicity, molecular weight, and molar volume are crucial properties for the sorption to MPs. The lower the hydrophobicity and molecular weight of a compound, the faster the diffusive mass transfer towards steady state or equilibrium (Müller et al. 2001; Pascall et al. 2005; Rochman et al. 2013). This implies that the diffusive mass transfer of, e.g., naphthalene $\left(\log K_{o w}=3.3, M W=128 \mathrm{~g} / \mathrm{mol}\right)$ will be faster than that of, e.g., benzo(a)pyrene $\left(\log K_{o w}=6.13\right.$, $\mathrm{MW}=252 \mathrm{~g} / \mathrm{mol})$. Furthermore, the planarity of the molecule influences how close it can move to the particle surface, thus facilitating adsorption (Velzeboer et al. 2014). 
Microplastics as contaminant vectors - exploring the processes - Postprint version

Desorption of adsorbed and absorbed molecules will depend on many factors and will generally decrease with increasing partition ratios and increasing binding strength. This is consistent with the general perception that absorbed molecules in amorphous organic matter desorb readily, whereas strongly adsorbed molecules are characterized as slowly desorbing or even desorption-resistant (Cornelissen et al. 2005; Mayer et al. 2011). It is also consistent with the wealth of studies showing that plastic additives, dissolved within the plastic matrix, can relatively easily leach out and into other media with sufficient solubilizing capacity. Even if seemingly counter-intuitive, these mechanisms suggest that desorption and leaching of HOCs from MPs often will occur to a greater extent and faster when they are absorbed rather than adsorbed.

The conditions of the surrounding environment, especially $\mathrm{pH}$, temperature, and ionic strength, can modulate plastic-contaminant interactions. Lower $\mathrm{pH}$ and higher temperature increase desorption of HOCs from MPs (Bakir et al. 2014). On the contrary, higher salinity increases the partitioning to the plastic polymer (Karapanagioti and Klontza 2008; Velzeboer et al. 2014). Furthermore, salinity influences the agglomeration/aggregation state of MPs which, in turn, can change properties such as total size and surface area (Velzeboer et al. 2014). It may be that these factors can result in differences in the sorption of HOCs to MPs between freshwater and marine environments and also cause differences in intra-organismal sorption/desorption behaviour compared to that occurring in the surrounding water.

\section{TRANSFER OF HYDROPHOBIC ORGANIC CHEMICALS BETWEEN MICROPLASTICS AND BIOTA}

A conceptual framework for mechanisms involved in the role of MPs as vectors for HOCs and their transfer into aquatic organisms has been proposed (Koelmans et al. 2016). This framework describes different scenarios whereby HOCs are released from MPs internally (after uptake) or externally (in the water phase or into natural food/prey), followed by uptake into the organism. An alternative model has been proposed by Bakir et al. (2016), including also considerations of 
differences in $\mathrm{pH}$, temperature, and the role of gut surfactants. What is not implicitly included in these model frameworks, however, is direct contact exposure. Here, we argue that these processes should not be overlooked in the evaluation of MPs as HOC vectors. In the organism's external environment, HOCs can be transferred from MPs to biota either via the aqueous phase or via direct contact exposure from MPs adhering to the exterior of the organism (i.e., the skin or exoskeleton) (see Figure 2). If MPs with sorbed HOCs are taken up by biota, HOCs can be transferred to the tissue either via intra-organismal fluids (e.g., gut fluid) or via direct contact exposure from MPs adhering to the interior of the organism (e.g., the gut or gill walls). Uptake via (pore) water and intra-organismal fluids requires desorption of the HOCs from the sorbent before uptake of the freely dissolved molecules. Desorption of molecules succeeded by uptake as freely dissolved molecules as well as external or internal direct contact exposure are (largely) governed by diffusion and partitioning.

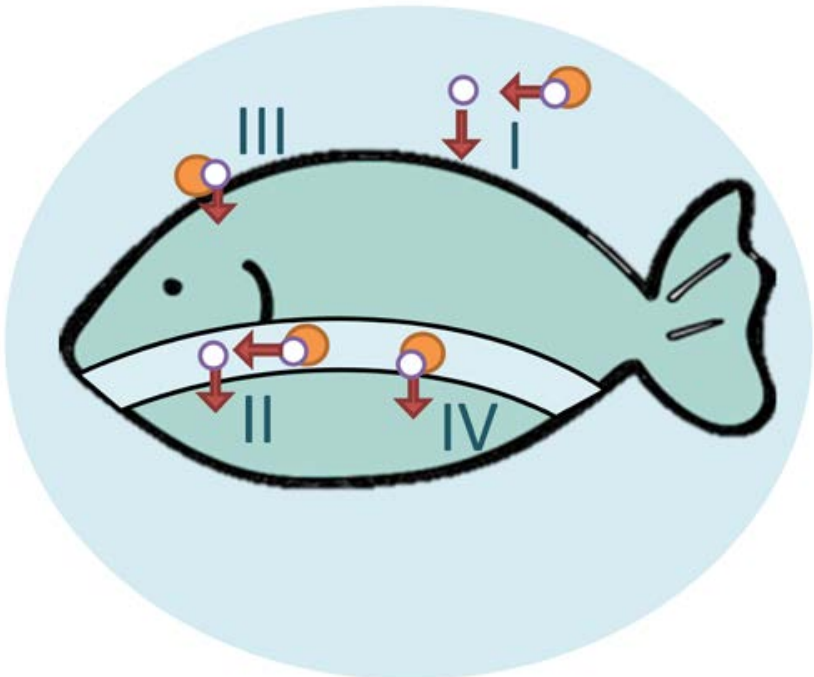

Figure 2. Four different diffusive mass transfer processes for HOCs between microplastics/naturally occurring sorbents and organisms: I) via water; II) via intra-organismal fluids; and, by direct contact exposure either III) external or IV) internal.

Diffusive mass transfer of a given HOC can differ greatly between various exposure media. Using a microscale experimental system with a clean and a PAH-loaded silicone disk, the diffusive mass transfer of PAHs was found to be much higher through the gut fluid of a sediment-dwelling worm than through water in 48-h experiments but the diffusive mass transfer was highest at direct contact between the disks (Mayer et al. 2007). HOCs sorbed to MPs are thus expected to be 
Microplastics as contaminant vectors - exploring the processes - Postprint version

transferred more rapidly to biota through digestive fluid than through water, and direct contact of MPs with exterior or interior parts of the organism might be an important and so far overlooked uptake route.

In natural environments, there are many phases that dissolve and bind HOCs to varying degrees, including water, dissolved organic carbon, colloid carbon, black carbon, natural fibres, bacteria, detritus biogenic silica (mainly diatoms), and other plankton (Koelmans et al. 2016; Dean et al. 1993; Ladewig et al. 2015). The processes described above also hold true for many other smallsized sorbents of HOCs in natural environments (Koelmans et al. 2016). It should however be noted that (de)sorption processes will differ widely between sorbents and HOCs, with partitioning being the governing process that not only determines the distribution of the HOCs between water and sorbent but that is also crucial for the release and bio-uptake of the chemicals.

The relative role of MPs as a vector for hazardous contaminants to organisms has been found negligible in comparison to natural exposure pathways (Gouin et al. 2011; Ziccardi et al. 2016; Koelmans et al. 2016; Bakir et al. 2016). This has mainly been attributed to the huge differences in mass between MPs and other sources. In oceans, the mass of water is estimated to be a factor of $10^{13}$ higher than the mass of plastic, and the volume of organic carbon in marine coastal environments was modelled to be more than $10^{7}$ times greater (Koelmans et al. 2016; Gouin et al. 2011). As a consequence, for example, it is predicted that sorption to PE would account for less than $0.1 \%$ of the HOCs in the oceans (Gouin et al. 2011). There may, however, be specific scenarios where MPs will play a larger role as a pathway for HOC transfer into biota than identified through such calculations (Bakir et al. 2016), as described below.

First, it is very likely that the actual occurrence of microplastics in the environment is currently underestimated due to sampling method issues (Kooi et al. 2016; Eerkes-Medrano et al. 2015) and a lack of consensus regarding sampling methods (Rocha-Santos and Duarte 2015). To illustrate the 
Microplastics as contaminant vectors - exploring the processes - Postprint version

importance of this issue, it was found that by decreasing the net mesh size, a substantial increase in numbers of collected particles was observed (Setälä et al. 2016). Quantification of MP occurrence is therefore highly influenced by the choice of sampling method. Second, the use and discard of plastics is increasing at a high rate resulting in an increased environmental occurrence of MPs (da Costa et al. 2016). In addition, specific local and small-scale conditions may apply. MPs are not evenly distributed in the environment and several studies have reported local hotspots, in which the fraction of MPs is much higher than in the oceans as a whole. This includes the North Pacific Gyre (Moore et al. 2001), industrial areas in the vicinity of plastic production plants (Norén 2007), and coastal sediments (Carson et al. 2011; Lee et al. 2015). On a local scale, MPs could therefore present a significant phase for the interaction with HOCs and subsequently for their transfer to organisms. Finally, to predict the role of MPs as vectors for HOCs it is important not only to understand the distribution of HOCs between different particulate matter, but also the ability of organisms to distinguish between natural food sources and MPs.

\section{LINKING LABORATORY AND ENVIRONMENTAL CONDITIONS}

The rapidly growing body of literature about the possible vector function of MPs can be roughly divided into two groups: (a) in situ studies reporting findings of MPs with associated contaminants in the environment (e.g. Endo et al. 2005; Llorca et al. 2014), and (b) laboratory experiments attempting to create robust experimental designs for studying vector effects and analysing the mechanisms and dynamics of MP-contaminant interactions and to measure biological effects (e.g., Hüffer \& Hofmann 2016; Lee et al. 2014; Browne et al. 2013).

Environmental sampling experiments provide a relatively unbiased view of the sorption of HOCs to MPs under given environmental conditions. However, directly linking cause and effects, for both chemical processes and biological effects, is challenged by the vast number of parameters that will have varied at a given location over time. Laboratory experiments, on the contrary, apply 
Microplastics as contaminant vectors - exploring the processes - Postprint version

conditions that may mimic, but not replicate, environmental conditions. They can be designed to give a clearer analysis of causality, but the duration of a laboratory experiment is often substantially shorter than the time scale during which MPs are present in the environment (Cole et al. 2015; Hall et al. 2015). Field sampling and laboratory testing are thus complementary. When exploring the role of MPs as HOC vectors in controlled laboratory studies, certain aspects of environmental realism can be included with regards to parameters that may be crucial to HOC sorption. This includes, for example, MP weathering prior to the test, test duration, variations in water chemistry, as well as performing tests in (synthetic) intra-organismal fluids.

\section{KNOWLEDGE GAPS AND FUTURE RESEARCH DIRECTIONS}

The mechanisms and kinetics of degradation of MPs, and the consequences for MPs as HOC vectors, are not well understood (Eerkes-Medrano et al. 2015; Rocha-Santos and Duarte 2015) and should thus be further investigated. Weathering of MPs should be considered in laboratory studies when evaluating HOC sorption and desorption, in addition to testing of pristine MPs. The processes of adsorption versus absorption of HOCs to MPs need to be better understood, especially with regards to how sorption and desorption kinetics may differ between MPs and other naturally occurring particulate matter, and how this may influence transfer into biota. Studies that directly correlate HOCs and MP uptake, thus implying a vector effect (Tanaka et al. 2013; Avio et al. 2015), are rare and no experimental studies were identified in the literature that demonstrated a vector effect followed by an explanation on a mechanistic or thermodynamic level. In particular, there seems to be a lack of understanding of the (thermo)dynamics of HOC desorption and translocation upon direct contact exposure (internally as well as externally). In order to measure these processes, it is necessary to better localize MPs and their associated HOCs inside organisms and tissues. However, for micro- and especially nano-sized particles, whose sizes are minute compared to the matrix they are in, this poses a range of challenges inherent to the related scientific field of nanotoxicology. It can thus be nearly impossible to localize and identify single particles in tissues 
Microplastics as contaminant vectors - exploring the processes - Postprint version

from exposed organisms (Jensen et al. 2016) even when fluorescent particles are used (Rosenkranz et al. 2009). Correlative studies employing fluorescence microscopy, electron microscopy, and nanoscale secondary ion mass spectrometry (NanoSIMS) to advance the knowledge of HOC transfer from MPs to organisms represent a new frontier. To more precisely assess the relative importance of MPs as vectors for HOCs, compared to natural pathways, there is a clear need for more appropriate sampling methods to avoid underestimations of actual environmental MP concentrations. Finally, selectivity for food-versus-plastic for different aquatic organisms should be further investigated and food webs, which are potentially vulnerable to MP facilitated HOC uptake, should be identified.

\section{CONCLUSION}

While the relative role of MPs as vectors for HOCs to organisms is generally considered minor in comparison to that of natural exposure pathways (such as water, food, and natural particulate matter) under present conditions, it is important to emphasize that: MP concentrations and environmental conditions change over time; and, spatiotemporal MP hotspots do (and will) occur. To better evaluate the role of MPs as pathways for HOC transfer into biota under such temporally and spatially varying conditions, an improved understanding of the governing processes is needed. For example, sorption/desorption processes differ between different polymers as well as between MPs and various natural particulate matter. Additionally, weathering will change HOC sorption and desorption. How these factors influence the role of MPs as HOC vectors is a topic of further research. Here, we have highlighted direct contact exposure as a route of HOC transfer from MPs into biota, a process which should not be overlooked when working towards a better understanding of MPs as HOC vectors in the environment. 
Microplastics as contaminant vectors - exploring the processes - Postprint version

\section{Acknowledgements}

We would like to thank the Technical University of Denmark for funding through the DTU-EPFL collaborative PhD grant of Sinja Rist.

Conflict of interest The authors declare no conflicts of interests. 


\section{References}

Avio CG, Gorbi S, Milan M, Benedetti M, Fattorini D, D’Errico G, Pauletto M, Bargelloni L, Regoli F. 2015. Pollutants bioavailability and toxicological risk from microplastics to marine mussels. Environ Pollut 198:211-222.

Bakir A, Rowland SJ, Thompson RC. 2014. Enhanced desorption of persistent organic pollutants from microplastics under simulated physiological conditions. Environ Pollut 185:16-23.

Bakir A, O’Connor IA, Rowland SJ, Hendriks AJ, Thompson RC. 2016. Relative importance of microplastics as a pathway for the transfer of hydrophobic organic chemicals to marine life. Environ. Pollut. 219:56-65.

Booij K, Robinson CD, Burgess RM, Mayer P, Roberts CA, Ahrens L, Allan IJ, Brant J, Jones L, Kraus UR, et al. 2016. passive sampling in regulatory chemical monitoring of nonpolar organic compounds in the aquatic environment. Environ Sci Technol 50:3-17.

Browne MA, Niven SJ, Galloway TS, Rowland SJ, Thompson RC. 2013. Microplastic moves pollutants and additives to worms, reducing functions linked to health and biodiversity. Curr Biol 23:2388-2392.

Carson HS, Colbert SL, Kaylor MJ, McDermid KJ. 2011. Small plastic debris changes water movement and heat transfer through beach sediments. Mar Pollut Bull 62:1708-1713.

Cole M, Lindeque P, Fileman E, Halsband C, Galloway TS. 2015. The impact of polystyrene microplastics on feeding, function and fecundity in the marine copepod Calanus helgolandicus. Environ Sci Technol 49:1130-1137.

Cornelissen G, Gustafsson Ö, Bucheli TD, Jonker MTO, Koelmans AA, Van Noort PCM. 2005. Extensive sorption of organic compounds to black carbon, coal, and kerogen in sediments and soils: Mechanisms and consequences for distribution, bioaccumulation, and biodegradation. Environ Sci Technol 39:6881-6895.

da Costa JP, Santos PSM, Duarte AC, Rocha-Santos T. 2016. (Nano)plastics in the environment Sources, fates and effects. Sci Total Environ 566-567:15-26.

Eerkes-Medrano D, Thompson RC, Aldridge DC. 2015. Microplastics in freshwater systems: A review of the emerging threats, identification of knowledge gaps and prioritisation of research needs. Water Res 75:63-82.

Endo S, Takizawa R, Okuda K, Takada H, Chiba K, Kanehiro H, Ogi H, Yamashita R, Date T. 2005. Concentration of polychlorinated biphenyls (PCBs) in beached resin pellets: Variability among individual particles and regional differences. Mar Pollut Bull 50:1103-1114.

Endo S, Yuyama M, Takada H. 2013. Desorption kinetics of hydrophobic organic contaminants from marine plastic pellets. Mar Pollut Bull 74:125-131.

Gouin T, Roche N, Lohmann R, Hodges G. 2011. A thermodynamic approach for assessing the environmental exposure of chemicals absorbed to microplastic. Environ Sci Technol 45:1466-72.

Hall NM, Berry KLE, Rintoul L, Hoogenboom MO. 2015. Microplastic ingestion by scleractinian corals. Mar. Biol. 162:725-732.

Hawthorne SB, Yang Y, Grabanski CB, Miller DJ, Lee ML. 2000. Response to comments on 
adsorption versus absorption of polychlorinated biphenyls onto solid-phase microextraction coatings. Anal Chem 72:642-643.

Hüffer T, Hofmann T. 2016. Sorption of non-polar organic compounds by micro-sized plastic particles in aqueous solution. Environ Pollut 214:194-201.

Jensen, L. H. S., Skjolding, L. M., Thit, A., Købler, C., Mølhave, K., Baun A. 2016. Not all that glitters is gold - an electron microscopy study on uptake of gold nanoparticles in Daphnia magna and related artifacts. Environ Chem (in press).

Karapanagioti HK, Klontza I. 2008. Testing phenanthrene distribution properties of virgin plastic pellets and plastic eroded pellets found on Lesvos island beaches (Greece). Mar Environ Res 65:283-290.

Koelmans AA, Bakir A, Burton GA, Janssen CR. 2016. Microplastic as a vector for chemicals in the aquatic environment: critical review and model-supported reinterpretation of empirical studies. Environ Sci Technol 50:3315-3326.

Lee H, Shim WJ, Kwon J-H. 2014. Sorption capacity of plastic debris for hydrophobic organic chemicals. Sci Total Environ 470-471:1545-1552.

Lee J, Lee JS, Jang YC, Hong SY, Shim WJ, Song YK, Hong SH, Jang M, Han GM, Kang D, et al. 2015. Distribution and size relationships of plastic marine debris on beaches in South Korea. Arch Environ Contam Toxicol 69:288-298.

Llorca M, Farré M, Karapanagioti HK, Barceló D. 2014. Levels and fate of perfluoroalkyl substances in beached plastic pellets and sediments collected from Greece. Mar Pollut Bull 87:28691.

Lohmann R. 2012. Critical review of low-density polyethylene's partitioning and diffusion coefficients for trace organic contaminants and implications for its use as a passive sampler. Environ Sci Technol 46:606-618.

Luthy RG, Aiken GR, Brusseau ML, Cunningham SD, Gschwend PM, Pignatello JJ, Reinhard M, Traina SJ, Weber WJ, Westall JC. 1997. Sequestration of hydrophobic organic contaminants by geosorbents. Environ Sci Technol 31:3341-3347.

Mato Y, Isobe T, Takada H, Kanehiro H, Ohtake C, Kaminuma T. 2001. Plastic resin pellets as a transport medium for toxic chemicals in the marine environment. Environ Sci Technol 35:318-324.

Mayer P, Vaes WHJ, Hermens JLM. 2000. Absorption of hydrophobic compounds into the poly(dimethylsiloxane) coating of solid-phase microextraction fibers: High partition coefficients and fluorescence microscopy images. Anal Chem 72:459-464.

Moore CJ, Moore SL, Leecaster MK, Weisberg SB. 2001. A Comparison of plastic and plankton in the North Pacific Central Gyre. Mar Pollut Bull 42:1297-1300.

Müller JF, Manomanii K, Mortimer MR, McLachlan MS. 2001. Partitioning of polycyclic aromatic hydrocarbons in the polyethylene/water system. Fresenius' J Anal Chem 371:816-822.

Norén F. 2007. Small plastic particles in coastal Swedish waters . KIMO Sweden. :1-11.

O’Connor IA, Golsteijn L, Hendriks AJ. 2016.Review of the partitioning of chemicals into different plastics: Consequences for the risk assessment of marine plastic debris. Mar Pollut Bull 113:17-24 
Pascall MA, Zabik ME, Zabik MJ, Hernandez RJ. 2005. Uptake of polychlorinated biphenyls (PCBs) from an aqueous medium by polyethylene, polyvinyl chloride, and polystyrene films. $J$ Agric Food Chem 53:164-169.

Rocha-Santos T, Duarte AC. 2015. A critical overview of the analytical approaches to the occurrence, the fate and the behavior of microplastics in the environment. TrAC Trends Anal Chem 65:47-53.

Rochman CM, Hoh E, Hentschel BT, Kaye S. 2013. Long-term field measurement of sorption of organic contaminants to five types of plastic pellets: Implications for plastic marine debris. Environ Sci Technol 47:1646-1654.

Rosenkranz P, Chaudhry Q, Stone V, Fernandes TF, Osenkranz PHR, Haudhry QAC, Tone VIS, Ernandes TEFF. 2009. A aomparison of nanoparticle and fine particle uptake by Daphnia Magna. Environ Toxicol Chem 28:2142-2149.

Rusina, TP, Smedes, F \& Klanova, J, 2010. Diffusion coefficients of polychlorinated biphenyls and polycyclic aromatic hydrocarbons in polydimethylsiloxane and low-density polyethylene polymers. J Appl Polym Sci 116:1803-1810.

Setälä O, Magnusson K, Lehtiniemi M, Norén F. 2016. Distribution and abundance of surface water microlitter in the Baltic Sea: A comparison of two sampling methods. Mar Pollut Bull 110:177183.

Tanaka K, Takada H, Yamashita R, Mizukawa K, Fukuwaka M, Watanuki Y. 2013. Accumulation of plastic-derived chemicals in tissues of seabirds ingesting marine plastics. Mar Pollut Bull 69:219-22.

Teuten EL, Saquing JM, Knappe DRU, Barlaz MA, Jonsson S, Bjorn A, Rowland SJ, Thompson RC, Galloway TS, Yamashita R, Ochi D, Watanuki Y, Moore C, Viet PH, Tana TS, Prudente M, Boonyatumanond R, Zakaria MP, Akkhavong K, Ogata Y, Hirai H, Iwasa S, Mizukawa K, Hagino Y, Imamura A, Saha M, Takada H 2009. Transport and release of chemicals from plastics to the environment and to wildlife. Philos Trans R Soc B Biol Sci 364:2027-2045.

Vaes WHJ, Mayer P, Oomen AG, Hermens JLM, Tolls J. 2000. Comments on “Adsorption versus absorption of polychlorinated biphenyls onto solid-phase microextraction coatings." Anal Chem 72:639-641.

Velzeboer I, Kwadijk CJAF, Koelmans AA. 2014. Strong sorption of PCBs to nanoplastics, microplastics, carbon nanotubes, and fullerenes. Environ Sci Technol 48:4869-4876.

Vethaak AD, Leslie HA. 2016. Plastic debris is a human health issue. Environ Sci Technol 50:6825-6826.

Ziccardi LM, Edgington A, Hentz K, Kulacki KJ, Kane Driscoll S. 2016. Microplastics as vectors for bioaccumulation of hydrophobic organic chemicals in the marine environment: A state-of-thescience review. Environ Toxicol Chem 35:1667-1676. 